\title{
Nonlinearity Analysis of a Short Channel CMOS Circuit for RFIC Applications
}

\author{
Yue $\mathrm{Wu}^{1,2}$, Hong-sun $\mathrm{Kim}^{1,2}$, Fredrik Jonsson ${ }^{2}$, Mohammed Ismail ${ }^{1,2}$, Håkan \\ Olsson $^{2}$ \\ ${ }^{1}$ Analog VLSI Lab, The Ohio State University \\ 205 Dreese Lab, 2015 Neil Ave. \\ Columbus, $\mathrm{OH}$ 43210, USA \\ ${ }^{2}$ Radio Electronics Lab, Royal Institute of Technology, \\ Electrum 229, S-164 40 Kista, Sweden \\ wuy, kimho, ismail@ee.eng.ohio-state.edu \\ fredrikj, hakan@ele.kth.se
}

\begin{abstract}
In this paper intermodulation phenomenon at Radio Frequency caused by the nonlinear characteristics of submicron MOS transistor has been analyzed. Common source stage with source degeneration is used for the analysis. The effects of bias current, transistor size and effective voltage on the 3rd order intermodulation(IM3) of the common source stage have been investigated. The analytical results are verified with APLAC simulations.
\end{abstract}

Keywords: Nonlinearity, Volterra Series, Low Noise Amplifier, Mixer, Intermodulation, OIP3

\section{INTRODUCTION}

Recently, great efforts have been spent on the integration of RF front-end circuits using CMOS/BiCMOS technologies. As the dimension of the transistors is scaling down continuously, the $f_{\max }$ of the transistors becomes large enough for typical RF applications(GSM, DECT, GPS, etc). Lots of papers [1]-[3] introduce the design methodology of Low Noise Amplifier (LNA) and downconversion mixer to achieve reasonable gain and good noise figure, but few of them mention the effect of the nonlinear characteristics of transistors in an analytical way. The reason is that it is very difficult to analyze the non-linear effects of circuits consisting of only a few transistors. Another reason is that 
even though transistor models nowadays are very accurate in the first order, higher order parameters, which determine the nonlinear characteristics of the transistors, are not modeled so accurately as the linear ones.

Common source stage with inductive source degeneration as shown in Fig.1 is widely used in RF front-end circuit. A detailed nonlinearity analysis of the bipolar case has been given in [4], but CMOS case has not been fully investigated yet. Since the CMOS technology has the advantage to integrate analog and digital parts together, it is very worthwhile to investigate CMOS nonlinear behavior in RF band. In this paper, nonlinearity analysis has been done on the circuit based on the short channel MOS model. In section II, the Volterra Kernals has been derived, then the effects of circuit elements' parameters on the Output Intercept Point( OIP3 ) have been addressed in section III. Finally sectionIV concludes this paper.

\section{DERIVATION OF VOLTERRA KERNALS}

Since in the case of LNA and downconversion mixer, the input signals are very small, the nonlinear behavior can be considered as weakly nonlinear, where it is enough to describe the nonlinearity with the first three orders of the input signal. In this case, Volterra Series is very suitable to predict the nonlinearity of the circuits. The circuit model we use is shown is Fig.2, $C_{g s}$ is the gate-source capacitance, which can be assumed as a linear device as long as the transistor works in saturation region[6]. $C_{g d}$ is neglected for simplicity. The output resistance $r_{o}$ of transistor can be neglected as long as the load impedance is small compared with $r_{o}$. The above assumption is pretty reasonable because when we consider the input stage's load of a cascode LNA or a Gilbert type mixer, the output load is about $1 / g_{m}$, which is small enough compared with $r_{o}$. $Z s, Z g$ are the source degeneration impedance and gate impedance respectively. $i_{D}$ is the drain current of the MOS transistor, which is the only nonlinear source in this analysis. For long channel devices, $i_{D}$ is modeled to have a square law relationship with $V_{\text {eff }}=V_{G S}-V_{t}$, but for short channel devices, this relationship is no longer valid. A simple equation which models the mobility degradation effect of short channel devices is as follows[6]:

$$
i_{D}=K \frac{\left(V_{e f f}+v_{g s}\right)^{2}}{1+\theta\left(V_{e f f}+v_{g s}\right)}
$$

where $K=\mu C_{o x} W / 2 L$, and $V_{e f f}=V_{G S}-V_{t}$.

Using Taylor series to expand this equation and removing the DC component from $i_{D}$, we get the AC signal current $i_{d}$ :

$$
i_{d}=i_{D}-I_{D}=g_{1} v_{g s}+g_{2} v_{g s}^{2}+g_{3} v_{g s}^{3}+\cdots
$$


where

$$
\begin{aligned}
g_{1} & =\frac{K V_{e f f}\left(2+\theta V_{e f f}\right)}{\left(1+\theta V_{e f f}\right)^{2}}, \\
g_{2} & =\frac{K}{\left(1+\theta V_{e f f}\right)^{3}}, \\
g_{3} & =\frac{-\theta K}{\left(1+\theta V_{e f f}\right)^{4}}
\end{aligned}
$$

There are several ways to calculate the Volterra Series, a direct method is as follows:

First we apply the Kirchoff Voltage Law(KVL) to the circuit of Fig.2, and we get:

$$
v_{s}=\left[s C_{g s}\left(Z_{g}+Z s\right)+1\right] v_{g s}+Z_{s} i_{d} .
$$

when we substitute eq. 2 into eq. 4 , we can find the nonlinear relationship between $v_{g s}$ and $v_{s}$ :

$$
\begin{aligned}
v_{s} & =\left[s C_{g s}\left(Z_{g}+Z s\right)+1\right] v_{g s}+Z_{s}\left[g_{1} v_{g s}+g_{2} v_{g s}^{2}+g_{3} v_{g s}^{3}\right] \\
& =a(s) v_{g s}+b(s) v_{g s}^{2}+c(s) v_{g s}^{3}
\end{aligned}
$$

where

$$
\begin{aligned}
a(s) & =s C_{g s}\left[Z_{g}(s)+Z_{s}(s)\right]+Z_{s}(s) g_{1}+1 \\
b(s) & =Z_{s}(s) g_{2} \\
c(s) & =Z_{s}(s) g_{3} .
\end{aligned}
$$

When we write the nonlinear transfer function of $i_{d}$ in terms of $v_{s}$,

$$
i_{d}=H_{1}\left(s_{1}\right) \circ v_{s}+H_{2}\left(s_{1}, s_{2}\right) \circ v_{s}^{2}+H_{3}\left(s_{1}, s_{2}, s_{3}\right) \circ v_{s}^{3}
$$

it is easy to find the desired Volterra Kernals are:

$$
\begin{aligned}
H_{1}\left(s_{1}\right)= & \frac{g_{1}}{\left.a_{(} s_{1}\right)} \\
H_{2}\left(s_{1}, s_{2}\right)= & \frac{H_{1}\left(s^{\prime \prime}\right) H_{1}\left(s_{1}\right) H_{1}\left(s_{2}\right)\left[g_{2} a\left(s^{\prime \prime}\right)-g_{1} b\left(s^{\prime \prime}\right)\right]}{g_{1}^{3}} \\
H_{3}\left(s_{1}, s_{2}, s_{3}\right)= & \frac{H_{1}\left(s^{\prime \prime \prime}\right) \gamma\left(s^{\prime \prime \prime}\right)}{g_{1}^{3}}\left[\frac{H_{1}\left(s_{1}\right) H_{1}\left(s_{2}\right) H_{1}\left(s_{3}\right)}{g_{1}} \times\right. \\
& \left.\left(g_{3}-\frac{2 g_{2}^{2}}{g_{1}}\right)+2 \overline{H_{1} H_{2}} g_{2}\right]
\end{aligned}
$$

where $s^{\prime \prime}=s_{1}+s_{2}, s^{\prime \prime \prime}=s_{1}+s_{2}+s_{3}$,

$$
\overline{H_{1} H_{2}}=\frac{H_{1}\left(s_{1}\right) H_{2}\left(s_{2}, s_{3}\right)+H_{1}\left(s_{2}\right) H_{2}\left(s_{1}, s_{3}\right)+H_{1}\left(s_{3}\right) H_{2}\left(s_{1}, s_{2}\right)}{3}
$$


and

$$
\gamma(s)=1+s C_{g s}\left[Z_{g}(s)+Z_{s}(s)\right] .
$$

So we have got the Volterra Kernals of $i_{d}$. From eq.8, we can calculate the first three orders of nonlinearity. In the next section, we will analyze the third order intermodulation using the above results.

\section{THIRD-ORDER INTERMODULATION}

Among those nonlinear effects, we are most interested in third order intermodulation components. This is because in communication systems, this component will block or desensitize the desired signal and cause significant degradation of system performance[5]. If in two-tone test with the input frequencies $\omega_{1}, \omega_{2}$ and equal amplitute $V_{s}$, the 3rd-order intermodulation at $2 \omega_{1}-\omega_{2}$ is defined as its amplitute ratio to the fundamental ones:

$$
I M 3=\frac{3}{4}\left|\frac{H_{3}\left(j \omega_{1}, j \omega_{1},-j \omega_{2}\right)}{H_{1}\left(j \omega_{1}\right)}\right| \cdot\left|V_{s}\right|^{2}
$$

Using eq. 8 , and since $\omega_{1} \approx \omega_{2} \approx \omega_{c}, \omega_{1}-\omega_{2} \ll \omega_{c}$, we have

$$
\begin{aligned}
I M 3= & \frac{3}{4}\left|V_{s}\right|^{2} \frac{\left|\gamma\left(s_{c}\right)\right|}{\left|\gamma\left(s_{c}\right)+Z_{s}\left(s_{c}\right) g_{1}\right|^{3}} \times \\
& \frac{1}{g_{1}}\left|g_{3}-\frac{2 g_{2}^{2}}{3}\left[2 B(\Delta s)+B\left(2 s_{c}\right)\right]\right|
\end{aligned}
$$

where $s_{c}=j \omega_{c}, \Delta s=j \omega_{2}-j \omega_{1}$, and $B(s)=\frac{Z_{s}(s)}{\gamma(s)+Z_{s}(s) g_{1}}$.

This is a very similar equation to the bipolar case. Since $\gamma$ appears in the nominator, we can get similar result as [4]: Using inductive degeneration will improve the linearity because it will generate a negative real value which can reduce the real part of $\gamma$, and it means that $|\gamma|$ can be reduced at some frequency. It is better than using resistive degeneration because of noise considerations, and capacitive degeneration will only degrade the linearity of the circuit.

Since $\Delta \omega$ is very small, $B(\Delta s)$ can be neglected because of the small inductance of the inductor at low frequency. Assuming $\gamma\left(2 s_{c}\right) \ll Z_{s}\left(2 s_{c}\right) g_{1}$, then $B\left(2 s_{c}\right) \approx 1 / g_{1}$. From eq.3, $g_{1}$ will increase with $V_{\text {eff }}$ while $g_{2}$ and $\left|g_{3}\right|$ will decrease. From eq.12, and note that $g_{3}$ has negative signs, increasing $V_{\text {eff }}$ will decrease IM3, which means improvement of linearity. Fig.4 shows the OIP3 vs biasing voltage using the theoretical result from eq. 12 and the result of APLAC simulation, the circuit used in simulation is shown in Fig. 3 and its parameters are $L_{s}=3 n H, L_{g}=6 n H, C_{g}=100 p f, C_{o x}=2.2 \mathrm{fF} / \mathrm{um}^{2}, f_{c}=$ $2 \mathrm{GHz}, \Delta f=5 \mathrm{MHz}$. We also show the relationship between OIP3 and the bias current in Fig.5, comparing with BJT[4], it can be found that given the same biasing current, MOS circuit is more linear than bipolar one as we expected. 
Since $g_{3}$ is a negative number, $g_{3}-\frac{2 g_{2}^{2}}{3}\left[2 B(\Delta s)+B\left(2 s_{c}\right)\right]$ is actually the summation of the two items. So, decreasing any of the following items will increase the circuit's linearity: $g_{2}, B\left(2 s_{c}\right), B(\Delta s)$ or $\left|g_{3}\right|$. If $\theta$ is very small(for long channel device), $g_{3}$ will decrease dramastically while $g_{1}$ and $g_{2}$ will not change much and the OIP3 will increase. This means that long channel device will be more linear. The comparision is also shown in Fig.4, where we choose $\theta=0.1$ and $\theta=0.5$ respectively for typical long channel and short channel devices, it can be seen that given the same size and biasing voltage, small $\theta$ will result in higher OIP3.

Eq. 3 also shows that $g_{1}, g_{2}$ and $g_{3}$ are all propotional to $K$, where $K=\mu C_{o x} W / 2 L$. According to the above assumption $B\left(2 s_{c}\right) \approx 1 / g_{1}, K$ can be cancelled in $\frac{1}{g_{1}}\left|g_{3}-\frac{2 g_{2}^{2}}{3}\left[2 B(\Delta s)+B\left(2 s_{c}\right)\right]\right|$, so the effect of $K$ on linearity is only determined by $g_{1}$ in $\frac{\left|\gamma\left(s_{c}\right)\right|}{\left|\gamma\left(s_{c}\right)+Z_{s}\left(s_{c}\right) g_{1}\right|^{3}}$, and IM3 will decrease when $K$ increase. So if we increase $W / L$ ratio, the OIP3 will increase, this can also be explained as the result of bias current increase, since we keep the bias voltage the same, then increase W/L means also the increase of bias current. Changing W/L of the transistor has another effect: tuning the gate source capacitance $C_{g s}$. Actually in RF front-end circuits, we always keep transistor's length at the smallest size, so we only consider the change of width. In most designs, the values of $L_{s}$ and $L_{g}$ are determined in order to cancel the imaginary part of the input impedance. The real part of the input impedance should be matched to the source impedance to obtain maximum power transfer. It is interesting to note that if we neglect the source impedance $R_{s}$ such resonation may null out the 3rd order intermodulation. From eq. 10 and eq.12, when the width is close the resonant size, $\gamma(s)$ will decrease till to be zero at the exact resonate size, so the OIP3 will be infinite, in other words, the third-order intermodulation componant is nulled out. Keep increasing the width, the magnitude of $\gamma(s)$ will begin to increase, this will cause the drop of OIP3. When W is large enough, the OIP3 increases with W because $g_{1}$ dominates eq.12. So the OIP3 will begin to increase again with $W$. When we consider source impedance $R_{s}$, we can calculate by inserting it into $Z_{g}(s), \gamma(s)$ then becomes $s C_{g s} R_{s}+1+s^{2} C_{g s}\left(L_{g}+L_{s}\right)$. At center frequency, $\gamma(s)$ will still have a remaining term $j \omega_{c} C_{g s} R_{s}$ which is not zero. Therefore the effect of 3rd-order nulling is not very significant. All these effects are shown in Fig.6 of APLAC simulation results. We can see from this figure how the OIP3 will change with the width of the transistor. We also notice that the smaller the $R_{s}$ is, the more nulling effect exists. We can also see that when $W$ is large enough, the slope of all those curves are almost the same, this matches with our expection because in that region, the dominating item $g_{1}$ are the same, the absolute value difference of OIP3 is caused by $\gamma(s)$ in the nominator of eq.12 of different $R_{s}$. The high peak occurs at about $W=485 \mu \mathrm{m}$, it is a little 
bit smaller than the theoretical size for resonation(about $520 \mu \mathrm{m}$ ), this can be explained by the Miller effect of $C_{g d}$ which we neglect in this analysis.

\section{CONCLUSION}

The nonlinear behavior of a special circuit has been analyzed, it is caused by the nonlinear characteristic of short channel MOS model. The Volterra Kernals have been used to derive the intermodulation expression, it is shown the linearity will increase with higher effective voltage or bias current, the size of transistor can have tuning effect on the linearity. The results have been verified by APLAC simulation with real $0.8 \mu \mathrm{m}$ CMOS process paramters.

\section{REFERENCES}

[1]A. R. Shahani, D. K. Shaeffer and T. H. Lee,"A 12-mW Wide Dynamic Range CMOS Front-End for a Portable GPS Receiver" it IEEE J. of Solidstate Circuits , vol. 32, no. 12, pp. 2061-2070, December, 1997.

[2]F. Piazza, Q. Huang, "A 1.57-GHz RF Front-End for Triple Conversion GPS Receiver", IEEE J. of Solid State Circuit, vol. 33, no. 2, pp. 202-209, Feb., 1998.

[3]K. L. Fong and R. G. Meyer,"A Class AB Monolithic Mixer for 900Mhz Applications," IEEE J. of Solid-state Circuits, vol. 32, no. 8, pp. 1166-1172, August, 1997.

[4]K. L. Fong and R. G. Meyer,"High Frequency Nonlinearity Analysis of Common-Emitter and Differential-Pair Transconductance Stages," IEEE J. of Solid-state Circuits, vol. 33, no. 4, pp. 548-555, April, 1998.

[5]R. G. Meyer and A. K. Wong,"Blocking and Desensitization in RF Amplifiers," IEEE J. of Solid-State Circuits, vol. 30, no. 8, pp. 944-946, August, 1995.

[6]Y. Tsividis, "Operation and modeling of the MOS transistor," McGraw-Hill, New York, 1987 


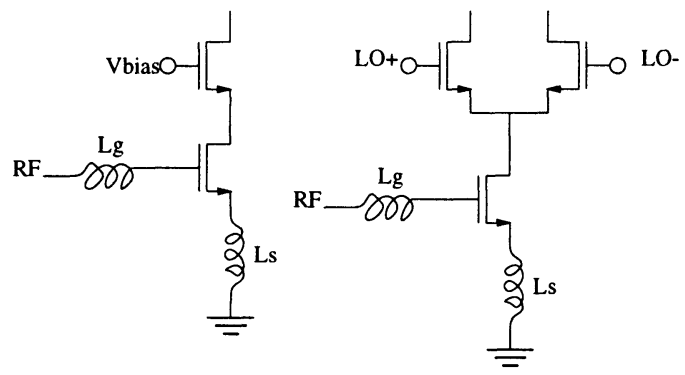

Figure 1 Common source circuit with degeneration in LNA and Mixer

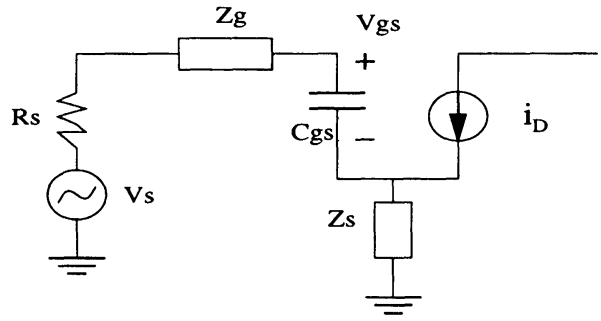

Figure 2 Circuit model used in analysis

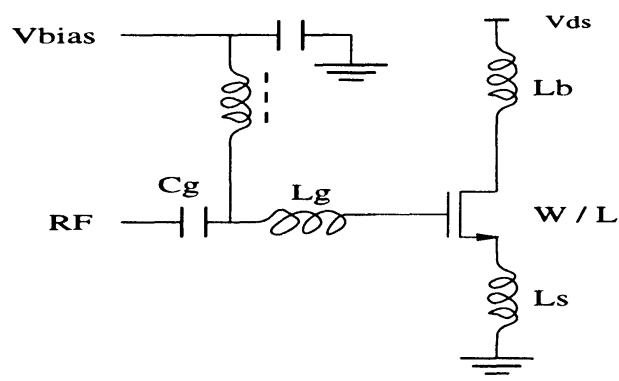

Figure 3 Circuit used in APLAC simulation 


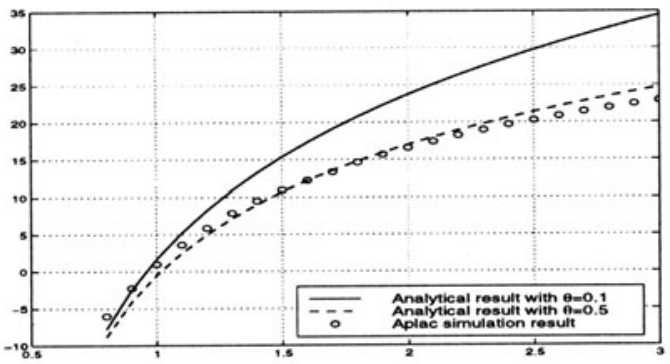

$V_{g s}$, analytical and APLAC results

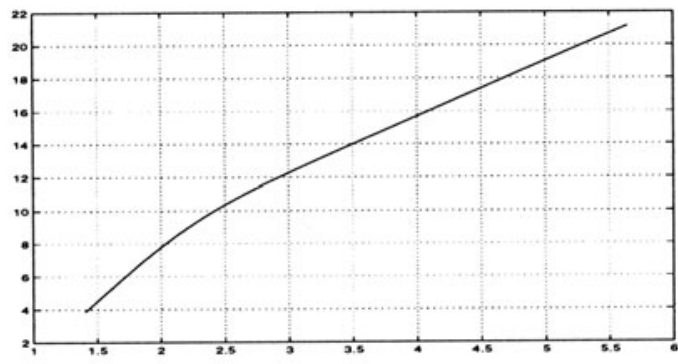

$I_{D}$

OIP3

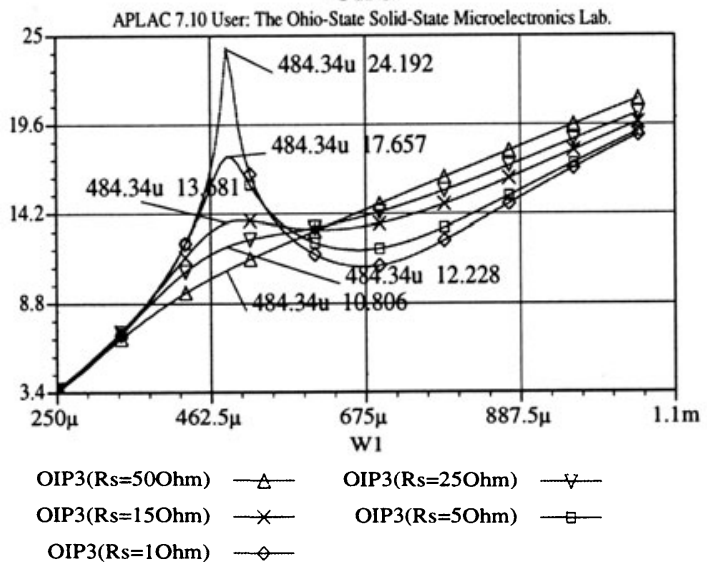

